\title{
Maximal independent systems of units in global function fields
}

\author{
by
}

Fei Xu (Hefei) and Jianqiang Zhao (Hefei and Providence, R.I.)

Introduction. Around 1980, Galovich and Rosen (cf. [GR1] and [GR2]) computed the index of cyclotomic units in the full group of units in a cyclotomic function field over a rational function field over a finite field. Later, Hayes [H1] and Oukhaba [O] obtained a few index formulae of the elliptic units in some special extensions of the global function fields with some restrictions on the infinite prime. Feng and Yin [FY] constructed the maximal independent systems of cyclotomic units in finite abelian extensions over a rational function field. Recently Shu [S] extended the result in [GR1] to the global function fields with degree one infinite prime. In the Mini-Conference on the Arithmetic of Function Fields held at Brown University in April 1996, Yin announced a result along this line which extended his work in $[Y]$ by removing the restriction on the degree of the infinite prime. This result seems to be the best one concerning the cyclotomic unit index in the sense that the base field can be any global function field. In this paper we construct maximal independent systems of units in an abelian extension over such a global function field.

Notations and terminology are standard if not explained. Specifically,

- $k$ : a global function field with a constant field $\mathbb{F}_{q}$ of $q$ elements.

- $\infty$ : a fixed infinite prime.

- $A$ : the ring of the functions in $k$ which are holomorphic away from $\infty$.

- $\mathbb{M}_{\infty}$ : the set of integral ideals of $A$.

- e: the unit ideal of $A$.

- $\mathbb{P}$ : the set of $k$-primes ( $k$-places).

- $k_{v}$ : the local field over $k$ completed at any $v \in \mathbb{P}$.

For any $\mathfrak{m} \in \mathbb{M}_{\infty}$,

- $\mathbb{P}_{\mathfrak{m}}:=\{\mathfrak{p} \in \mathbb{P}:(\mathfrak{p}, \mathfrak{m})=1\}$.

- $\mathbb{M}_{\mathfrak{m}}:=\left\{\mathfrak{b} \in \mathbb{M}_{\infty}:(\mathfrak{b}, \mathfrak{m})=1\right\}$.

- $\mathfrak{m}_{v}\left(\right.$ resp. $\left.A_{v}\right)$ : the completion of $\mathfrak{m}(\operatorname{resp} . A)$ in $k_{v}$. 
- $\Omega$ : the completion of the algebraic closure of $k_{\infty}$ at $\infty$.

- $v_{\infty}$ : the extension to $\Omega$ of the normalized valuation at $\infty$ on $k$.

- $K(\infty)$ : the constant field of $k_{\infty}$ which is a finite extension over $\mathbb{F}_{q}$ of degree $d_{\infty}$.

- $W_{\infty}:=q^{d_{\infty}}-1$ : the cardinality of $K(\infty)^{*}$.

- $\Phi(\mathfrak{a}):=\sharp(A / \mathfrak{a})^{*}$ for any $\mathfrak{a} \in \mathbb{M}_{\infty}$.

For any finite extension $E / k$,

- $O_{E}$ : the integral closure of $A$ in $E$.

- $h\left(O_{E}\right)$ : the ideal class number of $O_{E}$.

- $h(E)$ : the class number of $E$.

- $\mu(E)$ : the nonzero elements of the constant field of $E$.

- $W_{E}:=\sharp \mu(E)$.

- $E^{+}$: the maximal "real" subfield of $E$ which corresponds to the decomposition group of $\infty$ when $E$ is an abelian extension of $k$.

Now we briefly review some facts from rank one elliptic modules (cf. [H2]-[H4]). It is well known that there is a natural one-to-one correspondence between the set of isomorphism classes of rank one elliptic modules and the ideal classes of $A$ (see [H2] or [H4]). So there are exactly $h(A)$ isomorphism classes of rank one elliptic modules. In each class, we can choose a sign normalized (canonical) elliptic module $\varrho_{i}(i=1, \ldots, h(A))$ which is defined over $K_{\mathfrak{e}}$ (in fact $O_{K_{\mathfrak{e}}}$ ), where $K_{\mathfrak{m}}$ is by class field theory associated with the idèle subgroup $k^{*} U_{\mathfrak{m}, s}$ and

$U_{\mathfrak{m}, s}:=\left\{t=\left(t_{v}\right) \in J_{k}: s\left(t_{\infty}\right)=1, t_{v} \in A_{v}^{*}\right.$ for all $\left.v \neq \infty, t_{v} \equiv 1\left(\bmod \mathfrak{m}_{v}\right)\right\}$

for any $\mathfrak{m} \in \mathbb{M}_{\infty}$ and sign function $s$, where $J_{k}$ is the idèle group of $k$ (cf. [H2]-[H4]). Let $\Lambda_{\mathfrak{m}}^{(i)}$ be the set of $\mathfrak{m}$-torsions of $\varrho_{i}$, which is a cyclic $A$-module for any $\mathfrak{m} \in \mathbb{M}_{\infty}$. Then we can take its generator $\lambda_{\mathfrak{m}}=\xi(\mathfrak{m}) e_{\mathfrak{m}}(1)$ as defined by [H3, (5.5)], where $\xi(\mathfrak{m}) \in \Omega^{\times}$is chosen uniquely up to a factor of $K(\infty)^{*}$ to make $\xi(\mathfrak{m}) \mathfrak{m}$ correspond to a canonical elliptic module and $e_{\mathfrak{m}}(z)$ is the exponential function defined by

$$
e_{\mathfrak{m}}(z)=z \prod_{\gamma \in \mathfrak{m}-\{0\}}\left(1-\frac{z}{\gamma}\right), \quad \forall z \in \Omega .
$$

We know that $K_{\mathfrak{m}}=K_{\mathfrak{e}}\left(\Lambda_{\mathfrak{m}}^{(i)}\right)$ is an abelian extension over $k$ for $1 \leq i \leq$ $h(A)$. Let $H$ be the Hilbert class field of $k$, i.e., the maximal abelian unramified extension of $k$ in which $\infty$ splits completely (see $[\mathrm{R}]$ ) and $H_{\mathfrak{m}}=K_{\mathfrak{m}}^{+}$. Then

$\operatorname{Gal}\left(K_{\mathfrak{e}} / H\right) \cong K(\infty)^{*} / \mathbb{F}_{q}^{*}, \operatorname{Gal}\left(K_{\mathfrak{m}} / K_{\mathfrak{e}}\right) \cong(A / \mathfrak{m})^{*}, \operatorname{Gal}\left(K_{\mathfrak{m}} / H_{\mathfrak{m}}\right) \cong K(\infty)^{*}$.

From now on we always fix a sign function $s$. 
Maximal independent systems of units. Let $F$ be a subextension of $K_{\mathfrak{f}} / k$ for some $\mathfrak{f} \in \mathbb{M}_{\infty}$. Such an $\mathfrak{f}$ with least possible degree is called the conductor of $F$ and denoted by cond $(F)$. In fact, any finite abelian extension $F / k$ in which $\infty$ is tamely ramified must be contained in a constant field extension of some $K_{\mathfrak{f}}$ (cf. [H3, II]), so we can enlarge the constant field of $k$ to make things work. In this section, we will construct maximal independent systems of units in $F$.

Let $\widehat{G}$ denote the character group of any finite abelian group $G$. For any $\chi \in \operatorname{Gal} \widehat{(F} / k), \chi$ can be regarded as a map from $\mathbb{P}$ to $\mathbb{C}$ by Artin map. More precisely, let $I_{\mathfrak{p}}$ and $D_{\mathfrak{p}}$ denote the inertia group and the decomposition group of $\mathfrak{p}$ in $\operatorname{Gal}(F / k)$ respectively for any $\mathfrak{p} \in \mathbb{P}$. Therefore $D_{\mathfrak{p}} / I_{\mathfrak{p}}$ is a cyclic group generated by the Frobenius $\sigma_{\mathfrak{p}}$ of $\mathfrak{p}$. Then

$$
\chi(\mathfrak{p})= \begin{cases}\chi\left(\sigma_{\mathfrak{p}}\right) & \text { if } \chi\left(I_{\mathfrak{p}}\right)=1 \text { (unramified) } \\ 0 & \text { if } \chi\left(I_{\mathfrak{p}}\right) \neq 1 \text { (ramified) }\end{cases}
$$

So we can define the incomplete $L$-function

$$
L_{\mathfrak{n}}(s, \chi):=\prod_{\mathfrak{p} \in \mathbb{P}_{\mathfrak{n}}}\left(1-\chi(\mathfrak{p}) N \mathfrak{p}^{-s}\right)^{-1} \quad(s \in \mathbb{C}, \operatorname{Re}(s)>1)
$$

for any $\mathfrak{n} \in \mathbb{M}_{\infty}$, where $N \mathfrak{p}=q^{\text {deg } \mathfrak{p}}$ is the order of the residue class field at $\mathfrak{p}$. By our assumption on $F, W_{F}=W_{F^{+}}=q-1$ (see [H2]). Writing $L_{\mathfrak{e}}(s, \chi)=L(s, \chi)$, we have the following analytic class number formula (see $[\mathrm{W}])$ :

$$
h(F)=\frac{W_{F} h(k)}{q-1} \prod_{1 \neq \chi \in \operatorname{Gal}(F / k)} L(0, \chi)
$$

and $h(k) d_{\infty}=h(A)$.

Let $U$ (resp. $U^{+}$) be the group of units of $O_{F}$ (resp. $\left.O_{F+}\right)$. Then $\left[U: U^{+}\right]<\infty$ by the Dirichlet Unit Theorem (see [W]). Thus we only need to construct systems of units in $F^{+}$, which is what we will do essentially.

Let $\mathfrak{m} \in \mathbb{M}_{\infty}$ with $\mathfrak{f} \mid \mathfrak{m}$ and

$$
G_{\mathfrak{m}}=\operatorname{Gal}\left(H_{\mathfrak{m}} / k\right), \quad R=\operatorname{Gal}\left(K_{\mathfrak{m}} / F\right), \quad R^{+}=\operatorname{Gal}\left(H_{\mathfrak{m}} / F^{+}\right) .
$$

Let $r=\left[F^{+}: k\right]-1$ and $\sigma_{0}=\mathrm{id}, \sigma_{1}, \ldots, \sigma_{r} \in \operatorname{Gal}\left(K_{\mathfrak{m}} / k\right)$ be a set of representatives of

$$
\operatorname{Gal}\left(K_{\mathfrak{m}} / k\right) / R \operatorname{Gal}\left(K_{\mathfrak{m}} / H_{\mathfrak{m}}\right) \cong G_{\mathfrak{m}} / R^{+} \cong \operatorname{Gal}\left(F^{+} / k\right) .
$$

Suppose $\mathcal{D}_{0}=\left\{\mathfrak{a} \in \mathbb{M}_{\infty}: \mathfrak{e} \neq \mathfrak{a} \mid \mathfrak{m}\right\}, \mathcal{D}$ a nonempty subset of $\mathcal{D}_{0}$. For any $\mathfrak{a} \in \mathcal{D}$, let $\lambda_{\mathfrak{a}}=\xi(\mathfrak{a}) e_{\mathfrak{a}}(1)$ as in the introduction. Then we put

$$
\lambda_{i}:=N_{K_{\mathfrak{m}} / F} \prod_{\mathfrak{a} \in \mathcal{D}} \lambda_{\mathfrak{a}}^{\sigma_{i}-1} \quad(i=1, \ldots, r) .
$$


By [H3, Corollary 4.13 and Prop. 4.15], we have $\lambda_{i} \in U^{+}$and $\lambda_{i}$ will only differ up to an element in $\mu(F)=\mu\left(F^{+}\right)$by the different choices of $\sigma_{i}$ and $\lambda_{\mathfrak{a}}$. Therefore the following definition is correct.

Definition. $\mathfrak{C}=\mathfrak{C}(\mathfrak{m}, \mathcal{D}):=\mu\left(F^{+}\right)\left\langle\lambda_{1}, \ldots, \lambda_{r}\right\rangle$, the group generated by $\mu\left(F^{+}\right)$and $\lambda_{i}$ for $1 \leq i \leq r$.

It is obvious that $\mathfrak{C} \subseteq U^{+}$. In fact, we have

\section{THEOREM 1.}

$$
\left[U^{+}: \mathfrak{C}\right]=\frac{q-1}{W_{F^{+}}}\left(\frac{W_{\infty}}{e}\right)^{r} \frac{h\left(O_{F^{+}}\right)}{h(A)} i(\mathfrak{m}, \mathcal{D}),
$$

where

$$
i(\mathfrak{m}, \mathcal{D}):=\prod_{\substack{1 \neq \chi \in \widehat{G_{\mathfrak{m}}} \\ \chi\left(R^{+}\right)=1}} \sum_{\substack{\mathfrak{a} \in \mathcal{D} \text { such that } \\ \chi\left(\operatorname{Gal}\left(H_{\mathfrak{m}} / H_{\mathfrak{a}}\right)\right)=1}}\left[\frac{\Phi(\mathfrak{m})}{\Phi(\mathfrak{a})} \prod_{\mathfrak{q} \mid \mathfrak{a}}(1-\chi(\mathfrak{q}))\right],
$$

and $e=\left[F: F^{+}\right]$. Furthermore, $i(\mathfrak{m}, \mathcal{D})=0$ means that $\operatorname{rank}(\mathfrak{C})<$ $\operatorname{rank}\left(U^{+}\right)$.

Proof. Let $\beta$ be an infinite prime of $K_{\mathfrak{m}}$ above $\infty$, and $\beta^{+}, \gamma$ and $\gamma^{+}$ be the restrictions of $\beta$ in $H_{\mathfrak{m}}, F$ and $F^{+}$respectively. Let $D^{0}\left(F^{+}\right)$and $D^{0}\left(K_{\mathfrak{m}}\right)$ be the free abelian groups of degree zero divisors of $F^{+}$and $K_{\mathfrak{m}}$ generated by $\left\{\sigma_{i}\left(\gamma^{+}\right)-\gamma^{+}: 1 \leq i \leq r\right\}$ and $\left\{\sigma_{i}(\beta)-\beta: 1 \leq i \leq r\right\}$ respectively. Let $P\left(F^{+}\right)$and $P(\mathfrak{C})$ be the principal divisor groups generated by $\left\{\operatorname{div}(\alpha): \alpha \in U^{+}\right\}$and $\{\operatorname{div}(\alpha): \alpha \in \mathfrak{C}\}$ respectively. Since

$$
\left[U^{+}: \mathfrak{C}\right]=\left[P\left(F^{+}\right): P(\mathfrak{C})\right], \quad\left[D^{0}\left(F^{+}\right): P\left(F^{+}\right)\right]=h\left(F^{+}\right) d_{\infty} / h\left(O_{F^{+}}\right),
$$

we have

$$
\left[U^{+}: \mathfrak{C}\right]=\frac{h\left(O_{F^{+}}\right)}{h\left(F^{+}\right) d_{\infty}}\left[D^{0}\left(F^{+}\right): P(\mathfrak{C})\right]
$$

For any $\mathfrak{a} \in \mathcal{D}$, the infinite part of the principal divisor $\operatorname{div}\left(\lambda_{\mathfrak{a}}\right)$ in $K_{\mathfrak{m}}$ is

$$
W_{\infty} \sum_{\sigma \in \operatorname{Gal}\left(K_{\mathfrak{m}} / k\right) / \operatorname{Gal}\left(K_{\mathfrak{m}} / H_{\mathfrak{m}}\right)} v_{\infty}\left(\lambda_{\mathfrak{a}}^{\sigma}\right) \sigma^{-1}(\beta)
$$

since the ramification index of $\beta$ over $\infty$ is $W_{\infty}$. So

$$
\begin{aligned}
\operatorname{div}\left(\lambda_{\mathfrak{a}}^{\sigma_{i}-1}\right) & =W_{\infty} \sum_{\sigma \in \operatorname{Gal}\left(K_{\mathfrak{m}} / k\right) / \operatorname{Gal}\left(K_{\mathfrak{m}} / H_{\mathfrak{m}}\right)} v_{\infty}\left(\lambda_{\mathfrak{a}}^{\sigma}\right)\left(\sigma^{-1} \sigma_{i}(\beta)-\sigma^{-1}(\beta)\right) \\
& =W_{\infty} \sum_{\sigma \in \operatorname{Gal}\left(K_{\mathfrak{m}} / k\right) / \operatorname{Gal}\left(K_{\mathfrak{m}} / H_{\mathfrak{m}}\right)}\left[v_{\infty}\left(\lambda_{\mathfrak{a}}^{\sigma \sigma_{i}}\right)-v_{\infty}\left(\lambda_{\mathfrak{a}}^{\sigma}\right)\right] \\
& \times\left(\sigma^{-1}(\beta)-\beta\right) \in D^{0}\left(K_{\mathfrak{m}}\right) .
\end{aligned}
$$


Note that $N_{K_{\mathrm{m}} / F}(\beta)=\gamma$ and $\gamma^{+}=\gamma^{e}$ since $\left[F: F^{+}\right]=e$. We have

$$
\begin{aligned}
\operatorname{div}\left(\lambda_{i}\right)= & W_{\infty} \sum_{\mathfrak{a} \in \mathcal{D}} \sum_{\sigma \in \operatorname{Gal}\left(K_{\mathfrak{m}} / k\right) / \operatorname{Gal}\left(K_{\mathfrak{m}} / H_{\mathfrak{m}}\right)}\left[v_{\infty}\left(\lambda_{\mathfrak{a}}^{\sigma \sigma_{i}}\right)-v_{\infty}\left(\lambda_{\mathfrak{a}}^{\sigma}\right)\right]\left(\sigma^{-1}(\gamma)-\gamma\right) \\
= & \frac{1}{e} \sum_{\mathfrak{a} \in \mathcal{D}} \sum_{\sigma \in G_{\mathfrak{m}}}\left[v_{\infty}\left(\left(\lambda_{\mathfrak{a}}^{W_{\infty}}\right)^{\sigma \sigma_{i}}\right)-v_{\infty}\left(\left(\lambda_{\mathfrak{a}}^{W_{\infty}}\right)^{\sigma}\right)\right]\left(\sigma^{-1}\left(\gamma^{+}\right)-\gamma^{+}\right) \\
= & \frac{1}{e} \sum_{j=1}^{r} \sum_{\mathfrak{a} \in \mathcal{D}} \sum_{\sigma \in \sigma_{j} R^{+}}\left[v_{\infty}\left(\left(\lambda_{\mathfrak{a}}^{W_{\infty}}\right)^{\sigma \sigma_{i}}\right)-v_{\infty}\left(\left(\lambda_{\mathfrak{a}}^{W_{\infty}}\right)^{\sigma}\right)\right] \\
& \times\left(\sigma_{j}^{-1}\left(\gamma^{+}\right)-\gamma^{+}\right) \in D^{0}\left(F^{+}\right) .
\end{aligned}
$$

The last three lines make sense by [H3, Th. 4.17], $\left[K_{\mathfrak{a}}: H_{\mathfrak{a}}\right]=W_{\infty}$ and $H_{\mathfrak{a}} \subseteq H_{\mathfrak{m}}$ for any $\mathfrak{a} \in \mathcal{D}$. Therefore

$\left[D^{0}\left(F^{+}\right): P(\mathfrak{C})\right]<\infty \Leftrightarrow$

$\operatorname{det}\left[v_{\infty}\left(\left(\prod_{\mathfrak{a} \in \mathcal{D}} \prod_{\sigma \in R^{+}}\left(\lambda_{\mathfrak{a}}^{W_{\infty}}\right)^{\sigma}\right)^{\sigma_{i} \sigma_{j}}\right)-v_{\infty}\left(\left(\prod_{\mathfrak{a} \in \mathcal{D}} \prod_{\sigma \in R^{+}}\left(\lambda_{\mathfrak{a}}^{W_{\infty}}\right)^{\sigma}\right)^{\sigma_{i}}\right)\right]_{1 \leq i, j \leq r} \neq 0$

By the Dedekind determinant relation (see [S, Th. 1.5])

$$
\begin{aligned}
{\left[D^{0}\left(F^{+}\right): P(\mathfrak{C})\right] } & =e^{-r} \prod_{\substack{1 \neq \chi \in \widehat{G_{\mathfrak{m}}} \\
\chi\left(R^{+}\right)=1}} \sum_{j=1}^{r} \chi\left(\sigma_{j}\right) \sum_{\mathfrak{a} \in \mathcal{D}} \sum_{\sigma \in \sigma_{j} R^{+}} v_{\infty}\left(\left(\lambda_{\mathfrak{a}}^{W_{\infty}}\right)^{\sigma}\right) \\
& =e^{-r} \prod_{\substack{1 \neq \chi \in \widehat{G_{\mathfrak{m}}} \\
\chi\left(R^{+}\right)=1}} \sum_{\mathfrak{a} \in \mathcal{D}} \sum_{\sigma \in G_{\mathfrak{m}}} \chi(\sigma) v_{\infty}\left(\left(\lambda_{\mathfrak{a}}^{W_{\infty}}\right)^{\sigma}\right) .
\end{aligned}
$$

Now we set

$$
S(\mathfrak{m}, \mathfrak{a}, \chi)=\sum_{\sigma \in G_{\mathfrak{m}}} \chi(\sigma) v_{\infty}\left(\left(\lambda_{\mathfrak{a}}^{W_{\infty}}\right)^{\sigma}\right)
$$

Then

$$
S(\mathfrak{m}, \mathfrak{a}, \chi)= \begin{cases}\frac{\Phi(\mathfrak{m})}{\Phi(\mathfrak{a})} S\left(\mathfrak{a}, \mathfrak{a}, \chi_{\mathfrak{a}}\right) & \text { if } \chi\left(\operatorname{Gal}\left(H_{\mathfrak{m}} / H_{\mathfrak{a}}\right)\right)=1 \\ 0 & \text { if } \chi\left(\operatorname{Gal}\left(H_{\mathfrak{m}} / H_{\mathfrak{a}}\right)\right) \neq 1\end{cases}
$$

where $\chi_{\mathfrak{a}}=\left.\chi\right|_{\operatorname{Gal}\left(H_{\mathfrak{a}} / k\right)}$. So

$$
\begin{aligned}
{\left[D^{0}\left(F^{+}\right): P(\mathfrak{C})\right] } & =e^{-r} \prod_{\substack{1 \neq \chi \in \widehat{G_{\mathfrak{m}}} \\
\chi\left(R^{+}\right)=1}} \sum_{\mathfrak{a} \in \mathcal{D}} S(\mathfrak{m}, \mathfrak{a}, \chi) \\
& =e^{-r} \prod_{\substack{1 \neq \chi \in \widehat{G_{\mathfrak{m}}} \\
\chi\left(R^{+}\right)=1}} \sum_{\substack{\mathfrak{a} \in \mathcal{D} \text { such that } \\
\chi\left(\operatorname{Gal}\left(H_{\mathfrak{m}} / H_{\mathfrak{a}}\right)\right)=1}} \frac{\Phi(\mathfrak{m})}{\Phi(\mathfrak{a})} S\left(\mathfrak{a}, \mathfrak{a}, \chi_{\mathfrak{a}}\right) .
\end{aligned}
$$


By the last equation of [H3, P238] we have

$$
S\left(\mathfrak{a}, \mathfrak{a}, \chi_{\mathfrak{a}}\right)=W_{\infty} L_{\mathfrak{a}}\left(0, \chi_{\mathfrak{a}}\right)=W_{\infty} L(0, \chi) \prod_{\mathfrak{q} \mid \mathfrak{a}}(1-\chi(\mathfrak{q}))
$$

for any $\chi$ such that $\chi\left(H_{\mathfrak{m}} / H_{\mathfrak{a}}\right)=1$ and for any $\mathfrak{a} \in \mathcal{D}$. By the analytic class number formula (1) we have

$$
\left[U^{+}: \mathfrak{C}\right]=\frac{q-1}{W_{F^{+}}}\left(\frac{W_{\infty}}{e}\right)^{r} \frac{h\left(O_{F^{+}}\right)}{h(A)} i(\mathfrak{m}, \mathcal{D})
$$

where

$$
i(\mathfrak{m}, \mathcal{D})=\prod_{\substack{1 \neq \chi \in \widehat{G_{\mathfrak{m}}} \\ \chi\left(R^{+}\right)=1}} \sum_{\substack{\mathfrak{a} \in \mathcal{D} \text { such that } \\ \chi\left(\operatorname{Gal}\left(H_{\mathfrak{m}} / H_{\mathfrak{a}}\right)\right)=1}}\left[\frac{\Phi(\mathfrak{m})}{\Phi(\mathfrak{a})} \prod_{\mathfrak{q} \mid \mathfrak{a}}(1-\chi(\mathfrak{q}))\right] .
$$

Thus our theorem is proved.

Now we explain that $i(\mathfrak{m}, \mathcal{D}) \neq 0$ by suitable choice of $\mathfrak{m} \in \mathbb{M}_{\infty}$ and $\mathcal{D}$. First, we need a lemma.

LEMmA. Suppose $d$ and $n$ are positive integers and for any integer $t$ with $1 \leq t \leq d$,

$$
f_{t}\left(x_{1}, \ldots, x_{n}\right)=a_{t} \prod_{i=1}^{n}\left(x_{i}-b_{i, t}\right)-c \prod_{i=1}^{n}\left(x_{i}-1\right)
$$

is a $\mathbb{C}$-valued function defined on the set $U_{1} \times \ldots \times U_{n}=\left\{\left(x_{1}, \ldots, x_{n}\right)\right.$ : $\left.x_{i} \in U_{i}, 1 \leq i \leq n\right\}$, where each $U_{i}$ is a set of infinitely many integers which are different from one, $c$ is a nonzero integer, $a_{t}(1 \leq t \leq d)$ and $b_{j, t}$ $(1 \leq j \leq n, 1 \leq t \leq d)$ are given complex numbers. If for any $1 \leq t \leq d$ there is an integer $j(t)$ with $1 \leq j(t) \leq n$ such that $b_{j(t), t} \neq 1$, then there are infinitely many $\left(v_{1}, \ldots, v_{n}\right) \in U_{1} \times \ldots \times U_{n}$ such that

$$
\prod_{t=1}^{d} f_{t}\left(v_{1}, \ldots, v_{n}\right) \neq 0 \text {. }
$$

Pr o of. If $n=1$, then $f_{t}\left(x_{1}\right)$ is a nonzero linear form for $1 \leq t \leq d$ and the lemma is trivial.

For $n>1$, we can separate $f_{t}\left(x_{1}, \ldots, x_{n}\right)$ with $1 \leq t \leq d$ into two types.

Type I: If there is $\left(\alpha_{2}, \ldots, \alpha_{n}\right) \in U_{2} \times \ldots \times U_{n}$ such that

$$
f_{t}\left(x_{1}, \alpha_{2}, \ldots, \alpha_{n}\right)=0
$$

has infinitely many solutions in $U_{1}$ then

$$
f_{t}\left(x_{1}, \ldots, x_{n}\right)=\left(x_{1}-1\right) g_{t}\left(x_{2}, \ldots, x_{n}\right)
$$


where

$$
g_{t}\left(x_{2}, \ldots, x_{n}\right)=a_{t} \prod_{j=2}^{n}\left(x_{j}-b_{j, t}\right)-c \prod_{j=2}^{n}\left(x_{j}-1\right) .
$$

Type II: Otherwise, for any given $\left(\beta_{2}, \ldots, \beta_{n}\right) \in U_{2} \times \ldots \times U_{n}$,

$$
f_{t}\left(x_{1}, \beta_{2}, \ldots, \beta_{n}\right)=0
$$

has only finitely many solutions in $U_{1}$.

For all these $g_{t}$, there is $\left(\gamma_{2}, \ldots, \gamma_{n}\right) \in U_{2} \times \ldots \times U_{n}$ such that

$$
g_{t}\left(\gamma_{2}, \ldots, \gamma_{n}\right) \neq 0
$$

by induction on $n$. Note that

$$
f_{t}\left(x_{1}, \gamma_{2}, \ldots, \gamma_{n}\right)=0
$$

has finitely many solutions in $U_{1}$ for the $f_{t}\left(x_{1}, \ldots, x_{n}\right)$ of Type II and $U_{1}$ is the set with infinitely many elements. Hence there is $\gamma_{1} \in U_{1}$ such that

$$
f_{t}\left(\gamma_{1}, \gamma_{2}, \ldots, \gamma_{n}\right) \neq 0
$$

for all $1 \leq t \leq d$. Thus $\left(\gamma_{1}, \gamma_{2}, \ldots, \gamma_{n}\right)$ is as desired.

Take $U_{1}^{\prime}=U_{1} \backslash\left\{\gamma_{1}\right\}, \ldots, U_{n}^{\prime}=U_{n} \backslash\left\{\gamma_{n}\right\}$ as the above $U_{1}, \ldots, U_{n}$ and keep the above arguments, we are done.

For convenience in the following arguments, we assume $\mathcal{D}$ is the following special one:

$$
\mathcal{D}=\left\{\prod_{i \in I} \mathfrak{q}_{i}^{a_{i}}: I \subseteq L\right\}
$$

where $\mathfrak{m}=\prod_{i=1}^{l} \mathfrak{q}_{i}^{a_{i}}$ with $\mathfrak{q}_{i} \in \mathbb{P}$ and $L=\{1, \ldots, l\}$.

TheOREM 2. There are infinitely many $\mathfrak{m}$ such that

$$
\left[U^{+}: \mathfrak{C}(\mathfrak{m}, \mathcal{D})\right]<\infty .
$$

Proof. Write

$$
\mathfrak{f}=\operatorname{cond}(F)=\prod_{i=1}^{s} \mathfrak{q}^{a_{i}}
$$

with $\mathfrak{q}_{i} \in \mathbb{P}$ and $d+1=[F \cap H: k]$. Let

$$
\operatorname{Gal}(F \cap H / k)=\left\{\tau_{0}=1, \tau_{1}, \ldots, \tau_{d}\right\}
$$

and

$$
G(\widehat{F \cap H} / k)=\left\{\chi_{0}=1, \chi_{1}, \ldots, \chi_{d}\right\} .
$$

For each $1 \leq t \leq d$, put 


$$
a_{t}=\prod_{i=1}^{s}\left(\Phi\left(\mathfrak{q}_{i}^{a_{i}}\right)+1-\chi_{t}\left(\mathfrak{q}_{i}\right)\right)
$$

and

$$
f_{t}\left(x_{1}, \ldots, x_{d}\right)=a_{t} \prod_{j=1}^{d}\left(x_{j}-\chi_{t}\left(\tau_{j}\right)\right)-c \prod_{j=1}^{d}\left(x_{j}-1\right)
$$

where $c=\prod_{i=1}^{s} \Phi\left(\mathfrak{q}_{i}^{a_{i}}\right)=\Phi(\mathfrak{f})$. For $1 \leq t \leq d$, there are infinite prime ideals $\mathfrak{p}$ of $k$ such that every image of $\mathfrak{p}$ under the Artin map is $\tau_{t}, \Phi(\mathfrak{p})+1$ is not equal to one and all these $\mathfrak{p}$ 's form a set with infinitely many integers. Furthermore, there is $j(t)$ with $1 \leq j(t) \leq d$ such that $\chi_{t}\left(\tau_{j(t)}\right) \neq 1$ for each $1 \leq t \leq d$ since $\chi_{t} \neq 1$. Applying the above lemma, we have infinitely many $\left(\Phi\left(\mathfrak{p}_{1}\right)+1, \ldots, \Phi\left(\mathfrak{p}_{d}\right)+1\right)$ with $\operatorname{Frob}\left(\mathfrak{p}_{i}\right)=\tau_{i}, 1 \leq i \leq d$, such that

$$
\begin{aligned}
& \text { (2) } \prod_{t=1}^{d}\left[a_{t} \prod_{j=1}^{d}\left(\Phi\left(\mathfrak{p}_{j}\right)+1-\chi_{t}\left(\mathfrak{p}_{j}\right)\right)-c \prod_{j=1}^{d} \Phi\left(\mathfrak{p}_{j}\right)\right] \\
& =\prod_{t=1}^{d}\left[\prod_{i=1}^{s}\left(\Phi\left(\mathfrak{q}_{i}^{a_{i}}\right)+1-\chi_{t}\left(\mathfrak{q}_{i}\right)\right) \prod_{j=1}^{d}\left(\Phi\left(\mathfrak{p}_{j}\right)+1-\chi_{t}\left(\mathfrak{p}_{j}\right)\right)-\Phi\left(\mathfrak{f} \prod_{j=1}^{d} \mathfrak{p}_{j}\right)\right] \neq 0 .
\end{aligned}
$$

Put $\mathfrak{m}=\mathfrak{f} \prod_{i=1}^{d} \mathfrak{p}_{i}$. We claim this $\mathfrak{m}$ is as desired.

By Theorem 1 , we only need to show $i(\mathfrak{m}, \mathcal{D}) \neq 0$. Write $i(\mathfrak{m}, \mathcal{D})=i_{1} i_{2}$ where

$$
\begin{aligned}
& i_{1}= \prod_{\substack{1 \neq \chi \in \widehat{G_{\mathfrak{m}}}, \chi\left(R^{+}\right)=1 \\
\chi\left(\operatorname{Gal}\left(H_{\mathfrak{m}} / H\right)\right)=1}} \sum_{\mathfrak{a} \in \mathcal{D}} \frac{\Phi(\mathfrak{m})}{\Phi(\mathfrak{a})} \prod_{\mathfrak{q} \mid \mathfrak{a}}(1-\chi(\mathfrak{q})) \\
&= \prod_{\substack{1 \neq \chi \in \operatorname{Gal}(\widehat{H \cap F} / k) \\
=}} \frac{\Phi(\mathfrak{m})}{\Phi(\mathfrak{a})} \prod_{\mathfrak{q} \mid \mathfrak{a}}(1-\chi(\mathfrak{q})) \\
&=\prod_{1 \neq \chi \in \operatorname{Gal}(\widehat{H \cap F} / k)}\left[\prod_{i=1}^{s}\left(\Phi\left(\mathfrak{q}_{i}^{a_{i}}\right)+1-\chi\left(\mathfrak{q}_{i}\right)\right)\right. \\
&\left.\quad \times \prod_{j=1}^{d}\left(\Phi\left(\mathfrak{p}_{j}\right)+1-\chi\left(\mathfrak{p}_{j}\right)\right)-\Phi(\mathfrak{m})\right] \neq 0
\end{aligned}
$$

by (2) and

$$
i_{2}=\prod_{\substack{1 \neq \chi \in \widehat{G_{\mathfrak{m}}}, \chi\left(R^{+}\right)=1 \\ \chi\left(\operatorname{Gal}\left(H_{\mathfrak{m}} / H\right)\right) \neq 1}} \sum_{\substack{\mathfrak{a} \in \mathcal{D} \text { such that } \\ \chi\left(\operatorname{Gal}\left(H_{\mathfrak{m}} / H_{\mathfrak{a}}\right)\right)=1}} \frac{\Phi(\mathfrak{m})}{\Phi(\mathfrak{a})} \prod_{\mathfrak{q} \mid \mathfrak{a}}(1-\chi(\mathfrak{q}))
$$




$$
\begin{gathered}
=\prod_{\substack{1 \neq \chi \in \widehat{G_{\mathfrak{m}}}, \chi\left(R^{+}\right)=1 \\
\chi\left(\operatorname{Gal}\left(H_{\mathfrak{m}} / H\right)\right) \neq 1, \chi\left(\mathfrak{q}_{i}\right) \neq 0}}\left[\prod_{i=1}^{s}\left(\Phi\left(\mathfrak{q}_{i}^{a_{i}}\right)+1-\chi\left(\mathfrak{q}_{i}\right)\right)\right. \\
\left.\times \prod_{j=1}^{d}\left(\Phi\left(\mathfrak{p}_{j}\right)+1-\chi\left(\mathfrak{p}_{j}\right)\right)\right] \neq 0 .
\end{gathered}
$$

This completes the proof of Theorem 2 .

R e mark. In the above proof of Theorem $2, i_{1}$ comes from the unramified characters. This factor disappears if $k$ is a rational function field (see $[\mathrm{FY}]$ ). In order to construct maximal independent units in an abelian extension $F$ over a general global function field $k$, one has to construct enough units inside the Hilbert class field $H$ of $k$ or $H \cap F$ by the suitable choices of $\mathfrak{m}$. This fact was also pointed out in [S, Main Theorem 2, Theorem 5.2] explicitly in the very special situation.

Acknowledgments. Both authors would like to thank Professor K. Feng for introducing them into the theory of global function fields. They would also like to thank the referee for drawing their attention to $[\mathrm{S}]$ and pointing out some obscurities in the original version of this paper.

\section{References}

[FY] K. Feng and L. Yin, Maximal independent systems of units in cyclotomic function fields, Sci. China 34 (1991), 908-919.

[GR1] S. Galovich and M. Rosen, The class number of cyclotomic function fields, J. Number Theory 13 (1981), 363-375.

[GR2] - - - Units and class group in cyclotomic function fields, ibid. 14 (1982), 156184.

[H1] D. Hayes, Elliptic units in function fields, in: Proceedings of a Conference Related to Fermat's Last Theorem, D. Goldfeld (ed.), Birkhäuser, Boston, 1982, 321-341.

[H2 -, A brief introduction to Drinfeld modules, in: The Arithmetic of Function Fields, Proceedings of the Workshop at the Ohio State University, D. Goss, D. Hayes and M. Rosen (eds.), Walter de Gruyter, Berlin, New York, 1992, 1-32.

[H3] —, Stickelberger elements in function fields, Compositio Math. 55 (1985), 209239.

[H4] - Explicit class field theory in global function fields, in: Studies in Algebra and Number Theory, Adv. in Math. Suppl. Stud. 6, Academic Press, 1979, 173-271.

[O] H. Oukhaba, Elliptic units in global function fields, in: The Arithmetic of Function Fields, Proceedings of the Workshop at the Ohio State University, D. Goss, D. Hayes and M. Rosen (eds.), Walter de Gruyter, Berlin, New York, 1992, 87-102.

[R] M. Rosen, The Hilbert class field in function fields, Exposition. Math. 5 (1987), 365-378. 
[S] L. Shu, Class number formulas over global function fields, J. Number Theory 48 (1994), 133-161.

[W] A. Weil, Basic Number Theory, Springer, New York, 1974.

[Y] L. Yin, Index-class number formulas over global function fields, Preprint series 95-42, Department of Mathematics, University of Tokyo, 1995.

Department of Mathematics

University of Science and Technology of China

Hefei, Anhui 230026

P.R.China
Current address:

Department of Mathematics Brown University, Box 1917 Providence, Rhode Island 02912 U.S.A.

E-mail: jzhao@math.brown.edu

Received on 10.4.1995

and in revised form on 5.6.1996 